systems, each of which violates the like-numbered postulate and satisfies all the other postulates of the set. These examples are all arithmetic systems, the elements being the numbers 0 and 1 . The symbol $f(a, b)(\bmod 2)$ in an arithmetic system denotes the least positive residue modulo 2 obtained from $f(a, b)$ by rejecting multiples of 2 . The operations + and $X$ are to be interpreted as the operations of ordinary arithmetic when they occur in the modular expression, otherwise they are to be interpreted as logical addition and logical multiplication.

\begin{tabular}{|c|c|c|c|}
\hline EXAMPLE & $K$ & $a+b$ & $a b$ \\
\hline 1 & 0,1 & $a+b+0 / a b+1(\bmod 2)$ & $a b(\bmod 2)$ \\
2 & 0,1 & $a b(\bmod 2)$ & $a+b+0 / a b+1(\bmod 2)$ \\
3 & 0,1 & $a+b(\bmod 2)$ & $a b(\bmod 2)$ \\
4 & 0,1 & $a b(\bmod 2)$ & $a+b(\bmod 2)$ \\
5 & 0,1 & $a b(\bmod 2)$ & $a b(\bmod 2)$ \\
6 & Null & & \\
\hline
\end{tabular}

Paris, France

\title{
ON ANALYTIC FUNCTIONS WITH POSITIVE IMAGINARY PARTS
}

BY J. L. DOOB* AND B. O. KOOPMAN

The purpose of this paper is to give an integral representation of a function analytic in a half-plane, and with positive imaginary part there. This can be used to obtain in a simple way the well known analytic representation of the resolvent of a selfadjoint transformation in abstract Hilbert space.

Theorem. Let $\phi(l)$ be a function analytic for $\Im(l)>0$. If

$$
\Im[\phi(l)] \geqq 0, \quad \quad \quad \limsup |t \Im[\phi(i t)]|<\infty,
$$

for $t$ real, $t>0$, then there is a uniquely determined monotone non-decreasing function $\alpha(\lambda)$, defined for $-\infty<\lambda<\infty$, satisfying

* National Research Fellow.

$\dagger$ If $\xi$ is any complex number, $\Re(\xi)$ and $\Im(\xi)$ will be used to denote its real and imaginary parts, and $\bar{\xi}$ its conjugate. 


$$
\lim _{\lambda \rightarrow-\infty} \alpha(\lambda)=0, \quad \alpha(\lambda+0)=\alpha(\lambda),
$$

and

$$
\alpha(\lambda) \leqq \limsup _{t \rightarrow \infty}|t \Im[\phi(i t)]|, \quad(-\infty<\lambda<\infty),
$$

and such that

$$
\phi(l)=\int_{-\infty}^{\infty} \frac{d \alpha(\lambda)}{\lambda-l}+c,
$$

where $c$ is a real constant.*

The function

$$
\phi\left(-i \frac{z+1}{z-1}\right)
$$

is analytic for $|z|<1$ and $\Im(\phi) \geqq 0$ there. Then by a theorem of Herglotz, $\dagger$ there is a non-decreasing function $\alpha_{1}(\theta)$ defined for $0 \leqq \theta \leqq 2 \pi$, such that, if $z=r e^{i \psi}$,

(5) $\Im \phi\left(-i \frac{z+1}{z-1}\right)=\frac{1}{2 \pi} \int_{0}^{2 \pi} \frac{1-r^{2}}{1-2 r \cos (\theta-\psi)+r^{2}} d \alpha_{1}(\theta)$.

If $\alpha_{2}(\theta)=\alpha_{1}(\theta+0)-\alpha_{1}(0+0)$ for $0 \leqq \theta<2 \pi, \alpha_{2}(2 \pi)=\alpha_{1}(2 \pi-0)$ $-\alpha_{1}(0+0)$, this becomes

$$
\Im_{\phi}\left(-i \frac{z+1}{z-1}\right)=\frac{1}{2 \pi} \int_{0}^{2 \pi} \frac{1-r^{2}}{1-2 r \cos (\theta-\psi)+r^{2}} d \alpha_{2}(\theta)
$$

$$
+\frac{c_{1}}{2 \pi} \frac{1-r^{2}}{1-2 r \cos \psi+r^{2}}
$$

where $c_{1}=\alpha_{1}(2 \pi)-\alpha_{1}(2 \pi-0)+\alpha_{1}(0+0)-\alpha_{1}(0)$, and is evidently

* See results of R. Nevanlinna, Annales Academiae Scientiarum Fennicae, Series A, vol. 18 (1922), No. 5; W. Cauer, this Bulletin, vol. 38 (1932), p. 715, especially the footnote; Marshall Stone, Linear Transformations in Hilbert Space, Theorem 10.36; J. Wolff and F. de Kok, Bulletin de la Société Mathématique de France, vol. 50 (1932), pp. 221-227; S. Warschawski, Mathematische Annalen, vol. 107 (1932), pp. 1-15, who discusses integrals in the circle with the origin as center and of radius 1 which correspond to our integrals in the upper half-plane.

$\dagger$ Leipziger Berichte, Mathematische-Physikalische Klasse, vol. 63 (1911), pp. 508-511. 
not negative. Moreover the function $\alpha_{2}(\theta)$ is uniquely determined by $\Im(\phi)$; and $\alpha_{1}(\theta)$ is determined on a set of measure $2 \pi$, to within a constant,* which fixes $\alpha_{2}(\theta)$ uniquely. If we change $\theta, z$, to $\lambda$ and $l$, respectively, by

$$
\lambda=-i \frac{e^{i \theta}+1}{e^{i \theta}-1}=-\operatorname{ctn} \frac{\theta}{2}, \quad l=-i \frac{z+1}{z-1},
$$

(6) becomes, if $\alpha_{2}(\theta)=\alpha_{3}(\lambda)$,

$$
\Im[\phi(l)]=\frac{1}{\pi} \int_{-\infty}^{\infty} \frac{\Im(l)}{|\lambda-l|^{2}}\left(1+\lambda^{2}\right) d \alpha_{3}(\lambda)+\frac{c_{1}}{2 \pi} \Im(l) .
$$

Now if $l=i t, t>0$, this becomes

$$
\Im[\phi(i t)]=\frac{1}{\pi} \int_{-\infty}^{\infty} \frac{t}{\lambda^{2}+t^{2}}\left(1+\lambda^{2}\right) d \alpha_{3}(\lambda)+\frac{c_{1} t}{2 \pi},
$$

and it is seen at once that (1) implies that $c_{1}=0$ and that the integral $(1 / \pi) \int_{-\infty}^{\infty}\left(1+\lambda^{2}\right) d \alpha_{3}(\lambda)$ is finite and not greater than $\dagger$ $\lim \sup _{t \rightarrow \infty}|t \mathcal{F}[\phi(i t)]|$. If, then, $\alpha(\lambda)$ is defined by

$$
\alpha(\lambda)=\frac{1}{\pi} \int_{-\infty}^{\lambda}\left(1+\lambda^{2}\right) d \alpha_{3}(\lambda)
$$

it is seen to satisfy (2) and (3). Substituting in (7), we find

$$
\Im[\phi(l)]=\int_{-\infty}^{\infty} \frac{\Im(l)}{|\lambda-l|^{2}} d \alpha(\lambda)
$$

* For (5) is equivalent to

$$
\phi_{1}(z)=\frac{1}{i} \phi\left(-i \frac{z+1}{z-1}\right)=\frac{1}{2 \pi} \int_{0}^{2 \pi} \frac{e^{i \theta}+z}{e^{i \theta}-z} d \alpha_{1}(\theta)+i d,
$$

where $d$ is a real constant, from which it is seen at once that

$$
\phi_{1}{ }^{(n)}(0)=\frac{n !}{2 \pi} \int_{0}^{\pi} e^{-n i \theta} d \alpha_{1}(\theta), n \geqq 1, \Im \phi(i)=\frac{1}{2 \pi} \int_{0}^{2 \pi} d \alpha_{1}(\theta) .
$$

This determines $\alpha_{1}(\theta)$ to within a constant on a set of measure $2 \pi$, since it is a real function.

† This is the crucial step of the proof and since the same conclusions can be drawn if there is a sequence $\left\{\rho_{n} e^{i \tau_{n}}\right\},\left(0 \leqq \tau_{n}<\pi\right)$, such that $\lim _{\inf }{ }_{n \rightarrow \infty} \tau_{n}\left(\pi-\tau_{n}\right)$ $>0$, and such that lim $\sup _{n \rightarrow \infty}\left|\rho_{n} e^{i \tau_{n}} \Im\left[\phi\left(\rho_{n} e^{i \tau_{n}}\right)\right]\right|<\infty$, the lemma can be generalized accordingly. 
which is equivalent to

$$
\phi(l)=\int_{-\infty}^{\infty} \frac{d \alpha(\lambda)}{\lambda-l}+c,
$$

where $c$ is a real constant. The function $\alpha(\lambda)$, monotone nondecreasing and satisfying (2), is thereby uniquely determined. For otherwise, retracing steps, the fact that $\alpha_{2}(\theta)$ is uniquely determined would be contradicted. The representation (10) shows that unless $\phi(l) \equiv c, t \Im \phi(i t)$ increases monotonely with $t$ and that $\lim _{\lambda \rightarrow \infty} \alpha(\lambda)=\lim _{t \rightarrow \infty} t \Im[\phi(i t)]$.

CoROllaRy. If $\phi(l)$ is a function analytic for $\Im(l) \neq 0$ which can be put in the form (4) with $c=0$, where $\alpha(\lambda)$ is a complexvalued function satisfying (2), whose real and imaginary parts are of bounded variation over the interval $-\infty<\lambda<\infty$, then $\alpha(\lambda)$ is thereby uniquely determined.

This has been shown already (without supposing that $\phi(l)$ is defined for $\Im(l)<0)$ when $\alpha(\lambda)$ is supposed real and nondecreasing. The case when $\alpha(\lambda)$ is only supposed real can be reduced to this case, since any real function of bounded variation can be expressed as the difference between two monotone non-decreasing functions. In the general case we only have to show that $\phi(l) \equiv 0$ implies that $\alpha(\lambda) \equiv 0$. Suppose, then, that $\phi(l) \equiv 0$, and define $\phi_{1}(l), \phi_{2}(l)$ by

$$
\phi_{1}(l)=\int_{-\infty}^{\infty} \frac{d \Re \alpha(\lambda)}{\lambda-l}, \quad \phi_{2}(l)=\int_{-\infty}^{\infty} \frac{d \gamma_{\alpha}(\lambda)}{\lambda-l} .
$$

Then

$$
\phi_{1}(l)+i \phi_{2}(l) \equiv 0, \quad \Im(l) \neq 0 .
$$

Substituting $\bar{l}$ for $l, \phi_{1}(\bar{l})+i \phi_{2}(\bar{l}) \equiv 0, \Im(l) \neq 0$. But it is evident that $\phi_{j}(\bar{l})=\overline{\phi_{j}(l)},(j=1,2)$, so that $\overline{\phi_{1}(l)}+i \overline{\phi_{2}(l)} \equiv 0$, or, if we take the conjugate,

$$
\phi_{1}(l)-i \phi_{2}(l) \equiv 0, \quad \Im(l) \neq 0 .
$$

Equations (12) and (13) show that $\phi_{1}(l) \equiv \phi_{2}(l) \equiv 0$. We have already seen that this implies that $\Re \alpha(\lambda) \equiv \Im \alpha(\lambda) \equiv 0$, as was to be proved.

These results can be applied to the theory of self-adjoint 
transformations in Hilbert space as follows. Let $T$ be a selfadjoint transformation* in Hilbert space $\mathfrak{S}$, and let $R_{l}$ be the inverse of the transformation $T-l I$, where $\Im(l) \neq 0$, and $I$ is the identical transformation. Then if $f$ is an arbitrary element of $\mathfrak{S}$, $\left[R_{l} f, f\right]$ is analytic for $\Im(l) \neq 0$ and $\Im\left[R_{l} f, f\right]$ has the same sign $\dagger$ as $\Im(l)$. By the theorem proved in this paper there is then an integral representation of $\left[R_{l} f, f\right]$ :

$$
\left[R_{l} f, f\right]=\int_{-\infty}^{\infty} \frac{d \alpha(\lambda ; f)}{\lambda-l} .
$$

If $f$ and $g$ are arbitrary elements of $\mathfrak{S}$,

$$
\begin{aligned}
& {\left[R_{l} f, g\right]=\frac{1}{4}\left[R_{l}(f+g), f+g\right]-\frac{1}{4}\left[R_{l}(f-g), f-g\right]} \\
& +\frac{i}{4}\left[R_{l}(f+i g), f+i g\right]-\frac{i}{4}\left[R_{l}(f-i g), f-i g\right],
\end{aligned}
$$

which leads to an integral representation of $\left[R_{l} f, g\right]$,

$$
\left[R_{l} f, g\right]=\int_{-\infty}^{\infty} \frac{d \beta(\lambda ; f, g)}{\lambda-l},
$$

where $\beta(\lambda ; f, g)$ is a complex-valued function whose real and imaginary parts are of bounded variation over the infinite interval. It can then be shown that $\beta(\lambda ; f, g)=(E(\lambda) f, g)$, where the transformations $E(\lambda)$ form a resolution of the identity. $\neq$

Columbia University

* The notation used will be that in Stone, Linear Transformations in Hilbert Space, Colloquium Publications of this Society, vol. 15.

$\dagger$ Stone, loc. cit., Chapters 4 and 5.

$¥$ Carrying out the details of this would mean following the treatment given by Stone except that the considerations of Chapter $5, \S 2$, would be omitted, Theorem 5.4 being obtained directly from the theorem of this paper, as just explained. This treatment is similar in spirit to those given by Bochner, Sitzungsberichte der Preussischen Akademie der Wissenschaften, 1933, pp. 371-376, and by A. Khintchine, Proceedings of the National Academy of Sciences, vol. 19 (1933), pp. 567-573, who also separate the purely analytic facts out of certain results in Hilbert space. 